\title{
Tissue regeneration observed in a basic fibroblast growth factor-loaded porous acellular bovine pericardium populated with mesenchymal stem cells
}

Yen Chang, MD, ${ }^{a}$ Po-Hong Lai, PhD, ${ }^{\mathrm{b}}$ Hao-Ji Wei, MD, ${ }^{a}$ Wei-Wen Lin, MD, ${ }^{c}$ Chun-Hung Chen, MS, ${ }^{b}$ Shiaw-Min Hwang, PhD, ${ }^{d}$ Sung-Ching Chen, $\mathrm{PhD}^{\text {, }}$ and Hsing-Wen Sung, $\mathrm{PhD}^{\mathrm{b}}$

Supplemental material is available online.
From the Division of Cardiovascular Surgery, ${ }^{a}$ Veterans General Hospital-Taichung and College of Medicine, National YangMing University, Taipei, Taiwan, R.O.C.; Department of Chemical Engineering, ${ }^{\text {b }} \mathrm{Na}$ tional Tsing Hua University, Hsinchu, Taiwan, R.O.C.; Division of Cardiology, Veterans General Hospital-Taichung and Department of Life Science, Tunghai University, Taichung, Taiwan, R.O.C.; and Bioresource Collection and Research Center, ${ }^{\mathrm{d}}$ Food Industry Research and Development Institute, Hsinchu, Taiwan, R.O.C.

This work was supported by grants from the Ministry of Economic Affairs (94-EC17A-17S1-0009) and the National Health Research Institute (NHRI-EX95-9518EI), Taiwan, ROC.

Received for publication Nov 10, 2006; revisions received Feb 1, 2007; accepted for publication Feb 6, 2007.

Address for reprints: Professor Hsing-Wen Sung, PhD, Department of Chemical Engineering/Bioengineering Program, National Tsing Hua University, Hsinchu, Taiwan 30013 (E-mail: hwsung @ che.nthu.edu.tw).

J Thorac Cardiovasc Surg 2007;134:65-73

$0022-5223 / \$ 32.00$

Copyright $(92007$ by The American Association for Thoracic Surgery

doi:10.1016/j.jtcvs.2007.02.019
Objective: We sought to induce tissue regeneration within a porous patch for repair of a myocardial defect.

Methods: A basic fibroblast growth factor-loaded porous bovine pericardium populated with 5-bromo-2'-deoxyuridine-labeled mesenchymal stem cells was used as a cardiac patch (the basic fibroblast growth factor/mesenchymal stem cell patch) to repair a defect created in a syngeneic rat model. The blank porous pericardium (the control patch) and the patch loaded with basic fibroblast growth factor were used as controls. The implanted patches were retrieved at 4 and 12 weeks postoperatively $(\mathrm{n}=5$ per group at each time point).

Results: At retrieval, we found that none of the patches were thinned or dilated. Endothelialization and remesothelialization were observed on the endocardial and epicardial surfaces of patches in each of the studied groups, respectively. Additionally, newly regenerated muscle fibers, glycosaminoglycans, smooth muscle cells, and microvessels were seen in the middle layers of all patches, an indication of tissue regeneration. However, the extents of tissue regeneration in the basic fibroblast growth factor and basic fibroblast growth factor/mesenchymal stem cell patches were more pronounced than in those of the control patch. This may be attributed to the fact that the densities of neomicrovessels observed in the basic fibroblast growth factor and basic fibroblast growth factor/mesenchymal stem cell patches were significantly greater than in those of the control patch. 5-Bromo- $2^{\prime}$ deoxyuridine-labeled cardiomyocytes, smooth muscle cells, and endothelial cells were identified in the basic fibroblast growth factor/mesenchymal stem cells patch, and no cardiomyocytes were observed in the control and basic fibroblast growth factor patches.

Conclusion: The results provided evidence of tissue regeneration within a porous bovine pericardium through a process involving cell recruitment and tissue-specific differentiation.

$\mathrm{T}$ he human heart cannot regenerate significantly because adult cardiomyocytes are terminally differentiated and cannot replicate after injury. ${ }^{1} \mathrm{Re}-$ cently, the identification of stem cells capable of contributing to tissue regeneration has raised the possibility that cell therapy could be employed for repair of damaged myocardium. ${ }^{2}$ Bone marrow-derived mesenchymal stem cells (MSCs) retain the ability to differentiate into various types of tissue cells and contribute to the regeneration of a variety of mesenchymal tissues including bone, cartilage, muscle, and adipose. ${ }^{3,4}$ Several studies performed on rodents, pigs, and human demonstrated that MSCs have the potency to differentiate into a cardiomyocyte phenotype in the heart. ${ }^{5,6}$ 

Abbreviations and Acronyms
5 -aza $=5$-azacytidine
$\alpha$-SMA $=\alpha$-smooth muscle actin
$\mathrm{bFGF}=$ basic fibroblast growth factor
BrdU = 5-bromo-2'-deoxyuridine
$\mathrm{ECM}=$ extracellular matrix
EVG = elastic van Gieson
GAG = glycosaminoglycan
$\mathrm{H} \& \mathrm{E}=$ hematoxylin and eosin
MSC = mesenchymal stem cell
SEM = scanning electron microscopic
SMC $=$ smooth muscle cell

products, Taichung, Taiwan) aqueous solution (phosphate-buffered saline solution, $\mathrm{pH} 7.4)$ at $37^{\circ} \mathrm{C}$ for 3 days. The denaturation temperature $\left(74.8^{\circ} \mathrm{C} \pm 0.2^{\circ} \mathrm{C}\right)$, fixation index $(58.4 \% \pm 4.7 \%)$, pore size $(159.8 \pm 26.7 \mu \mathrm{m})$, and porosity $(94.9 \% \pm 1.7 \%)$ of the fixed porous acellular tissues $(n=5)$ were previously reported. $^{15}$

In the present study, to facilitate cell infiltration and repopulation, the dense layer on each side of the fixed porous acellular tissues was further sliced off using a cryostat microtome. After preparation of test samples, the porous acellular tissues (the control patch) were processed for scanning electron microscopic (SEM) examinations to investigate their ultrastructures. The obtained samples were then sterilized in a graded series of ethanol solutions for loading bFGF and populating MSCs in vitro and for the subsequent animal study.

In our previous study, an acellular bovine pericardium with a porous structure was used to repair a myocardial defect. ${ }^{7}$ Smooth muscle cells together with neomuscle fibers were clearly identified in the porous acellular patch, an indication of tissue regeneration. However, cardiomyocytes were not found within the patch, a significant limitation of this study. Additionally, the amount of neomicrovessels observed in the porous acellular patch at retrieval was significantly less than that observed in the native myocardium. Vascularization in extracellular matrices (ECMs) to support the metabolic needs of the engineered tissues is generally a prerequisite for achieving appropriate tissue regeneration and function. ${ }^{8}$ Investigations have incorporated angiogenic factors such as basic fibroblast growth factor (bFGF) to stimulate angiogenesis in tissueengineering ECMs. ${ }^{9}$

In the present study, to enhance both vascularization and tissue regeneration, a bFGF-loaded porous bovine pericardium populated with MSCs was used as a cardiac patch to repair a surgically created myocardial defect in the right ventricle of rats.

\section{Materials and Methods}

\section{Isolation, Culture, and Differentiation of MSCs}

Bone marrow MSCs were isolated from femora and tibia of Lewis rats as described previously. ${ }^{10}$ To induce myogenic differentiation, the DNA-demethylating agent 5-azacytidine (5-aza) was added on the third day and incubated with MSCs for 24 hours. Subsequently, the induced MSCs were labeled in vitro for later identification by adding $100 \mu \mathrm{g} / \mathrm{mL}$ 5-bromo-2'-deoxyuridine (BrdU)containing media to $50 \%$ confluent cultures for 24 hours. ${ }^{11}$ After 7-day induction, the cultured cells were immunohistochemically stained for cardiac-specific troponin $\mathrm{T}$ and BrdU. ${ }^{12}$ Samples $(\mathrm{n}=$ 5) were analyzed using a FACScan flow cytometer (Becton Dickinson, San Jose, Calif).

\section{Porous Acellular Bovine Pericardia (Control Patch)}

Details of the methodology used to prepare porous acellular bovine pericardia were previously reported. ${ }^{13,14}$ The obtained porous acellular tissues were fixed in a $0.05 \%$ genipin (Challenge Bio-

\section{bFGF-loaded Acellular Tissues (bFGF Patch)}

The sterilized acellular tissues were freeze-dried under aseptic conditions and then loaded with bFGF using a method described in the literature. ${ }^{15}$ Briefly, solutions of human recombinant bFGF at different known concentrations buffered with phosphate-buffered saline solution were sterilized using a $0.22-\mu \mathrm{m}$ filter. Subsequently, the aforementioned freeze-dried samples were rehydrated in the prepared bFGF solutions overnight (the bFGF patch). The amount of bFGF loaded in the patch was determined by measuring the difference between the initial and residual amounts of bFGF in the solution using a sandwich enzyme-linked immunosorbent assay $(\mathrm{n}=5)$.

\section{bFGF-loaded Acellular Tissues Populated With MSCs (bFGF/MSC Patch)}

After the process of 5-aza induction and BrdU labeling (for 2 days), MSCs were washed, harvested from culture dishes using $0.05 \%$ trypsin solution, and resuspended to a concentration of $1 \times$ $10^{6}$ cells in $50 \mu \mathrm{L}$ of culture medium containing $1250 \mathrm{ng}$ bFGF. Similar to preparation for the bFGF patch, this medium was then used to rehydrate the freeze-dried acellular tissues at $37^{\circ} \mathrm{C}$ overnight (the bFGF/MSC patch). To investigate cell attachment and distribution on the bFGF/MSC patch, after 18-hour incubation, samples were fixed in $10 \%$ phosphate-buffered formalin for 2 days and subsequently processed for histologic and SEM examinations.

\section{Animal Study}

Animal care and use were in compliance with the "Guide for the Care and Use of Laboratory Animals" prepared by the Institute of Laboratory Animal Resources, National Research Council, and published by the National Academy Press, revised 1996. Adult syngeneic Lewis rats weighing 250 to $275 \mathrm{~g}$ were used for the right ventricular wall replacement. The prepared patches (control, bFGF, or bFGF/MSC patches, in a disk shape with a diameter of $5 \mathrm{~mm}$ and a thickness of $1.6 \mathrm{~mm}$ ) were used to repair a transmural defect surgically created in the right ventricle of syngeneic rat hearts based on a method reported by Ozawa and colleagues. ${ }^{16}$

\section{Epicardial Electrograms}

A 12-lead electrocardiographic system (PC-ECG 1200M, Norav Medical, Kiryat Bialik, Israel) was used to acquire the epicardial 
electrograms of the implanted patch and its adjacent rat native myocardium immediately after implantation and at retrieval. ${ }^{17}$

\section{Echocardiography}

Cardiac ultrasonography was performed with a commercially available echocardiographic system (SONOS 5500, Agilent Technologies, Andover, Mass) equipped with a $12-\mathrm{MHz}$ broadband sector transducer before patch implantation and at retrieval. The heart was imaged in the 2-dimensional mode in short-axis views at the midpapillary level of the left ventricle to evaluate contractions of the patch-implanted myocardium at the right ventricle. ${ }^{18}$

\section{Histologic Examinations}

The implanted patches were retrieved at 4 and 12 weeks postoperatively ( $\mathrm{n}=5$ per group at each time point) and were used for gross and histologic examinations. The samples used for light microscopy were fixed in $10 \%$ phosphate-buffered formalin, embedded in paraffin, sectioned into a thickness of $5 \mu \mathrm{m}$, and then stained with hematoxylin and eosin (H\&E). Also, sections of test samples were stained with Masson's trichrome or elastic van Gieson (EVG) for the detection of collagen fibrils and muscle fibers or stained with safranin-O to visualize glycosaminoglycans (GAGs). Additional sections were stained with a van Gieson solution to visualize mesothelial cells. ${ }^{19}$

Immunohistochemical staining with a monoclonal antibody against BrdU was used to identify the cells populated in the bFGF/MSC patch, revealed by a peroxidase-antiperoxidase technique. ${ }^{10}$ Additionally, sections of the retrieved patches were stained with a monoclonal antibody against $\alpha$-sacromeric actin (clone 5C5, Serotec, Kidlington, Oxford, UK). ${ }^{5}$

A monoclonal antibody against $\alpha$-smooth muscle actin $(\alpha$ SMA, DAKO Corp., Carpinteria, Calif) was used to identify smooth muscle cells (SMCs). Additional sections were stained for factor VIII with an immunohistologic technique with a monoclonal anti-factor VIII antibody (DAKO). ${ }^{20}$ The density of neomicrovessels in each studied sample was quantified with a computer-based image analysis system (Image-Pro Plus, Media Cybernetics, Silver Spring, Md) and converted to vessels $/ \mathrm{mm}^{2}$. Five different microscopic fields ( $\times 100$ by ECLIPSE-E800, Nikon, Tokyo, Japan) of each patch portion of the right ventricular wall were randomly selected. $^{10}$

\section{Statistical Analysis}

Statistical analysis for the determination of differences in the measured properties between groups was accomplished using 1-way analysis of variance and determination of confidence intervals, which was performed with a computer statistical program (Statistical Analysis System, Version 6.08, SAS Institute Inc., Cary, NC). All data are presented as mean values \pm standard deviations.

\section{Results}

\section{Phenotype of MSCs Before Implantation}

Morphology of the isolated cells initially appeared small and rounded with a tendency to grow in clusters. Nonadherent cells were removed by medium change at 24 hours and every 4 days thereafter. Elongated cells with a spindle- shaped morphology appeared after 72 hours and reached confluence after 10 to 14 days. Seven days after 5-aza treatment, flow cytometric analysis of the 5-aza-induced MSCs demonstrated a significant difference in the expression of troponin $\mathrm{T}$ as compared with the control group (Figure E1). The nuclei of the cells induced by 5-aza were labeled with BrdU for 24 hours pretransplantation. It was found that $97.6 \% \pm 1.2 \%$ of the culture cells stained positively $(\mathrm{n}=5)$.

\section{Test Patches}

After fixation by genipin, the color of acellular bovine tissues turned into bluish. A dense layer was present on each side of the acellular bovine pericardia (Figure 1, a). After slicing off these dense layers with a cryostat microtome, a porous structure beneath was revealed (the control patch, Figure $1, b$ and $d$ ). It was found that the highest amount of bFGF that could be loaded in porous acellular bovine pericardia was approximately $500 \pm 15 \mathrm{ng}$ per sample (a 5 -mm disk with a thickness of $1.6 \mathrm{~mm}, \mathrm{n}=5$ ). This specific patch (the bFGF patch) was used for the animal study. Results of the in vitro study revealed that after an initial burst, bFGF was released gradually in a sustained manner. After cell populating, MSCs were mostly present in the surface layers of porous acellular tissues and had a uniform, viable spindleshaped morphology (18 hours after cell seeding, the bFGF/ MSC patch contained the same amount of bFGF as in the bFGF patch; Figure 1, $c$ and $e$ ). BrdU incorporation was evident in $>90 \%$ of the populated MSCs (Figure $1, f$ ).

\section{Epicardial Electrograms}

Immediately after implantation, no epicardial electrogram signals were observed in the studied groups. At 4 weeks postoperatively, the mapping of the electrograms on each of the studied group showed low amplitudes as compared with that observed on its adjacent native myocardium. The amplitudes of local electrograms on the epicardial surfaces of all studied groups increased significantly with increasing the implantation duration. However, the amplitudes of the electrograms observed on the $\mathrm{bFGF}$ and $\mathrm{bFGF/MSC}$ patches were significantly stronger than those on the control patch throughout the entire course of the study.

\section{Echocardiography}

Short-axis 2-dimensional images of the normal heart as well as the control patch-implanted heart, the bFGF patchimplanted heart, and the bFGF/MSC patch-implanted heart at retrieval were obtained using a commercially available echocardiographic system. The results indicated that all studied groups were akinetic. The differences in the global contractions at the right ventricles for all patch-implanted hearts were difficult to differentiate when compared with the normal heart. 


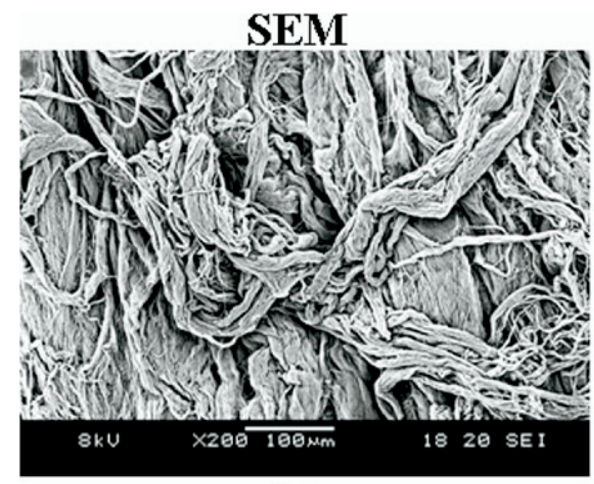

(a)

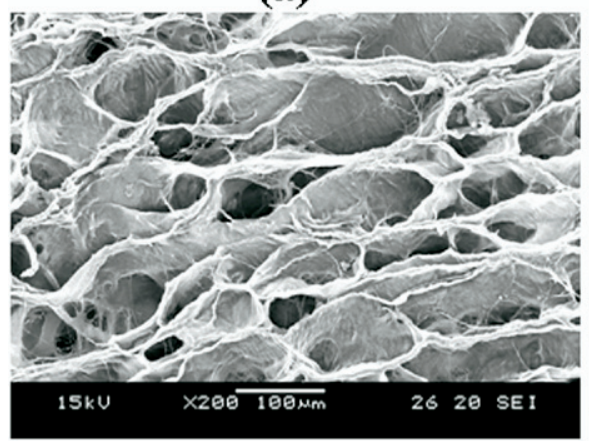

(b)

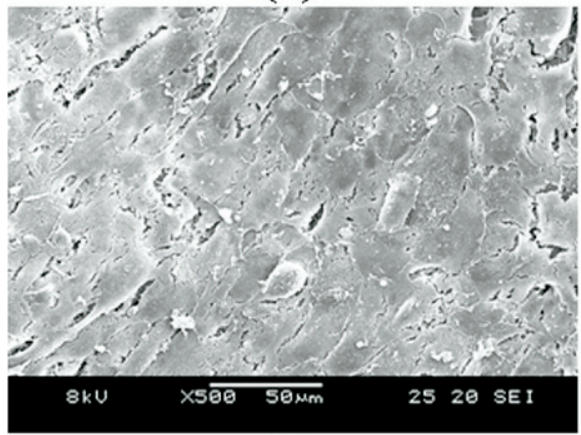

(c)

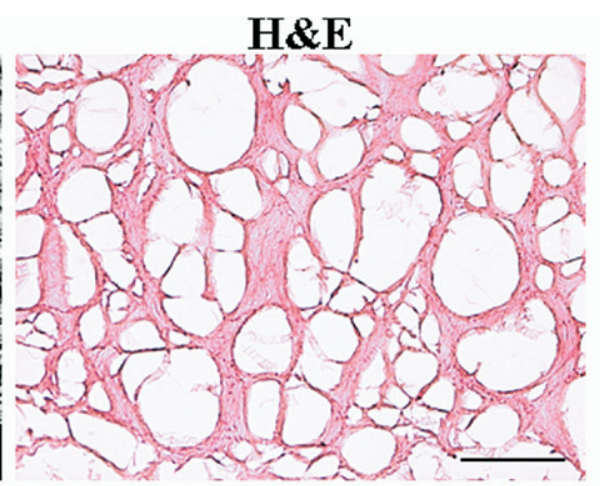

(d)

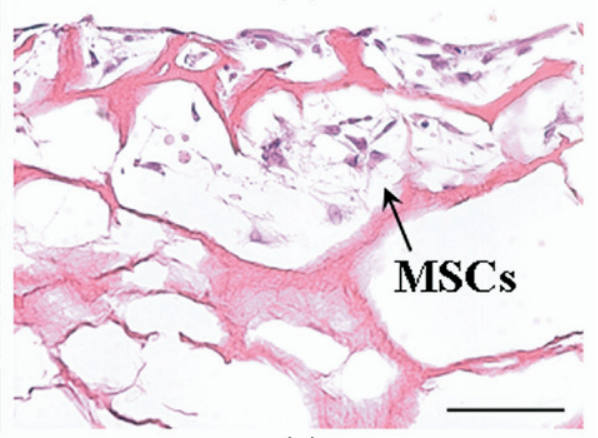

(e)

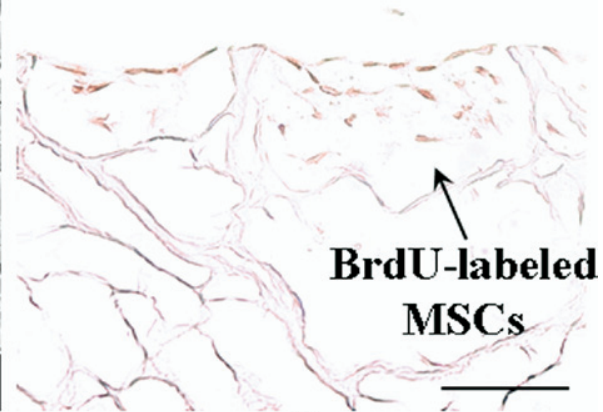

Figure 1. Ultrastructures of a porous acellular bovine pericardium (a) before and (b) after slicing off the dense layers on its surfaces using a cryostat microtome; (c) SEM micrograph of the bFGF/MSC patch, 18 hours after cell seeding; (d) photomicrograph of a porous acellular bovine pericardium (the control patch) before implantation stained with H\&E; (e) photomicrograph of the bFGF/MSC patch before implantation stained with H\&E; (f) photomicrograph of the $\mathrm{bFGF/MSC}$ patch stained with a monoclonal antibody against BrdU. Scale bars, $100 \mu \mathrm{m}$ (d) and $50 \mu \mathrm{m}$ (e and f).

\section{Gross Examination}

On the inner (endocardial) surface, intimal thickening was observed for all studied groups; however, no thrombus formation was found (Figure E2). No apparent tissue adhesion was observed on the outer (epicardial) surface of any of the patches throughout the entire course of the study. Additionally, no aneurysmal dilation was seen in any of the studied groups.

\section{Histologic Findings}

At 4 weeks postoperatively, host cells together with neoconnective tissue fibrils and neomicrovessels were clearly observed to fill the pores within the inner and outer layers of patches from all studied groups, an indication of tissue regeneration (Figure E3). The neoconnective tissue fibrils regenerated in the $\mathrm{bFGF}$ and $\mathrm{bFGF} / \mathrm{MSC}$ patches were more compact and organized than control patch samples. Factor VIII-positive endothelial cells were visible on the inner surfaces of patches from all studied groups, and mesothelial cells, positively stained with van Gieson, were observed on their outer surfaces (Figure 2).

Tissue regeneration was also observed to fill most of the pores in the middle layers of the bFGF and bFGF/MSC patches, although there were still some unfilled pores present in the control patch. The neoconnective tissue fibrils regenerated in these studied patches were identified to be neomuscle fibers (stained red) with some neocollagen fibrils (stained blue), as determined with Masson's trichrome stain (Figure 3). The identified neomuscle fibers were further 

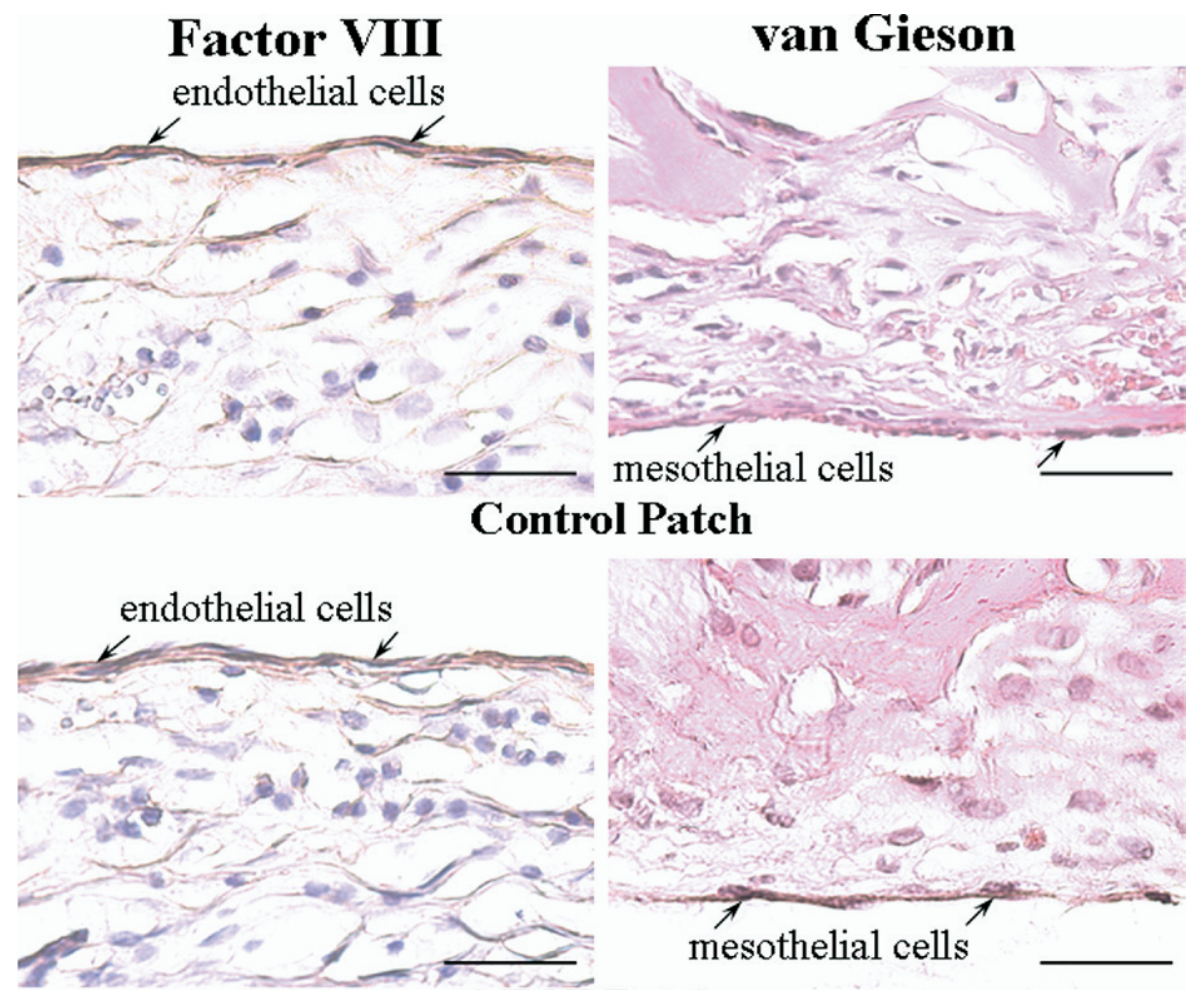

\section{bFGF Patch}

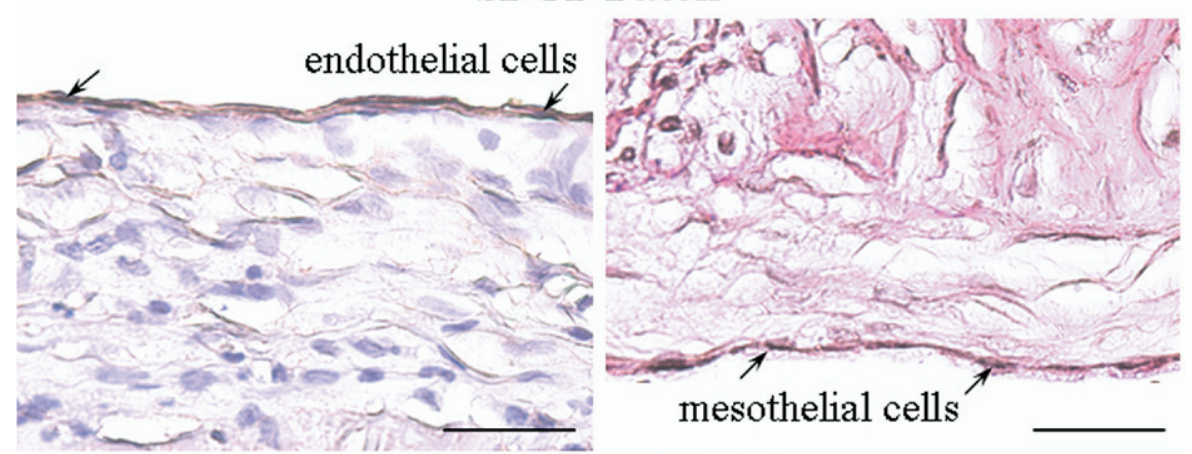

bFGF/MSC Patch
Figure 2. Photomicrographs of the inner (endocardial) and outer (epicardial) surfaces of the control, bFGF, and bFGF/MSC patches retrieved at 4 weeks postoperatively stained for factor VIII and with van Gieson, respectively. Scale bars, $30 \mu \mathrm{m}$. confirmed by the EVG stain. Additionally, there were some GAGs regenerated within the pores of each studied patch. Moreover, $\alpha$-SMA positively stained cells were seen in patches from all studied groups (Figure 3), indicating the presence of SMCs.

The densities of neomicrovessels found in the bFGF $\left(462 \pm 29\right.$ vessels $\left./ \mathrm{mm}^{2}\right)$ and bFGF/MSC patches $(489 \pm 36$ vessels $/ \mathrm{mm}^{2}$ ) were significantly greater than that observed in the control patch $\left(189 \pm 37\right.$ vessels $\left./ \mathrm{mm}^{2}\right)$, and that in the normal/adjacent myocardium was about $1161 \pm 75$ vessels/ $\mathrm{mm}^{2}$. Additionally, BrdU-labeled cells were clearly identified in the bFGF/MSC patch (Figure E4). Some of the identified BrdU-labeled cells were further stained positively for $\alpha$-sacromeric actin, indicating that a portion of the implanted MSCs had been differentiated toward the car- diomyogenic lineage and expressed cytoplasmic $\alpha$-sacromeric actin. Moreover, a few capillary walls composed of BrdUlabeled endothelial cells and some BrdU-labeled SMCs were recognized in the bFGF/MSC patch. The BrdUlabeled cardiomyocytes, SMCs, and endothelial cells observed in the bFGF/MSC patch were about $32 \pm 3,74 \pm 5$, and $17 \pm 3$ cells $/ \mathrm{mm}^{2}$, respectively. In contrast, no cardiomyocytes were observed in the control and bFGF patches.

At 12 weeks postoperatively (Figure 4), the neoconnective tissue fibrils seen in all studied groups were comparatively more compact and organized than their counterparts observed at 4 weeks postoperatively. The density of neomicrovessels observed in the control patch $(187 \pm 23$ vessels/ $\mathrm{mm}^{2}$ ) did not significantly increase with time, whereas those observed in the bFGF $\left(524 \pm 18\right.$ vessels $\left./ \mathrm{mm}^{2}\right)$ and $\mathrm{bFGF} /$ 


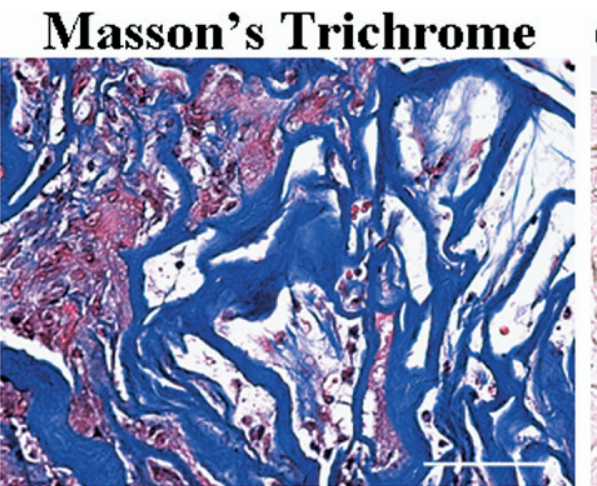

\section{$\alpha$-Smooth Muscle Actin}

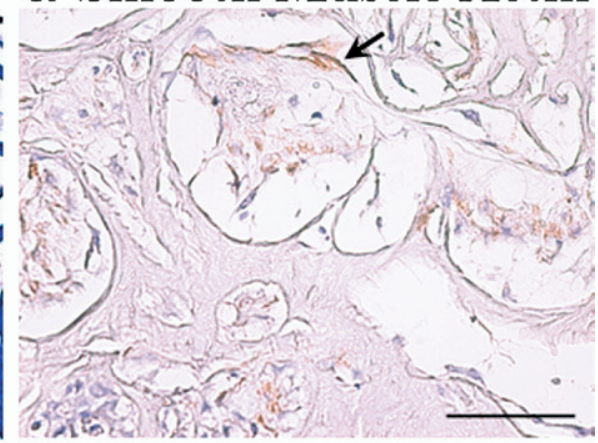

\section{Control Patch}
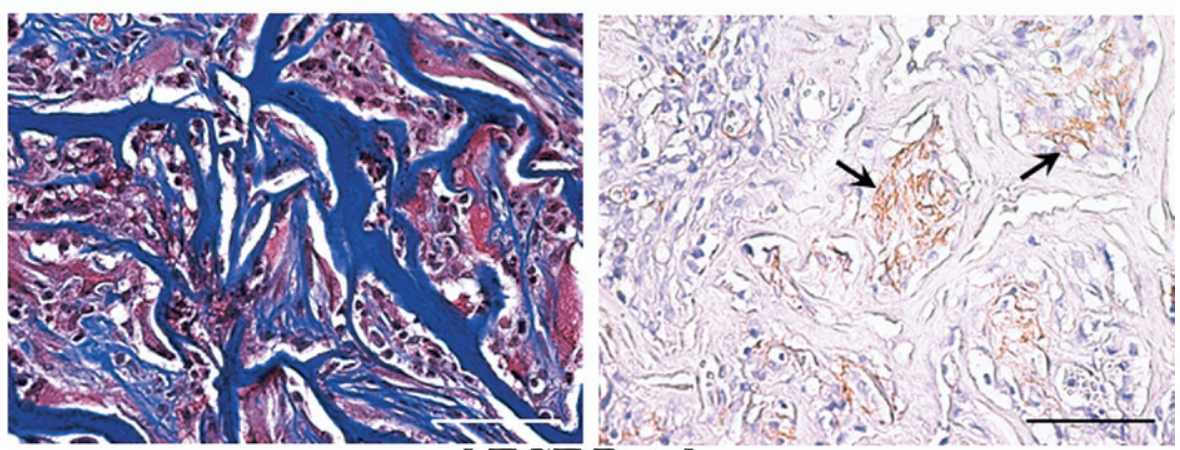

bFGF Patch
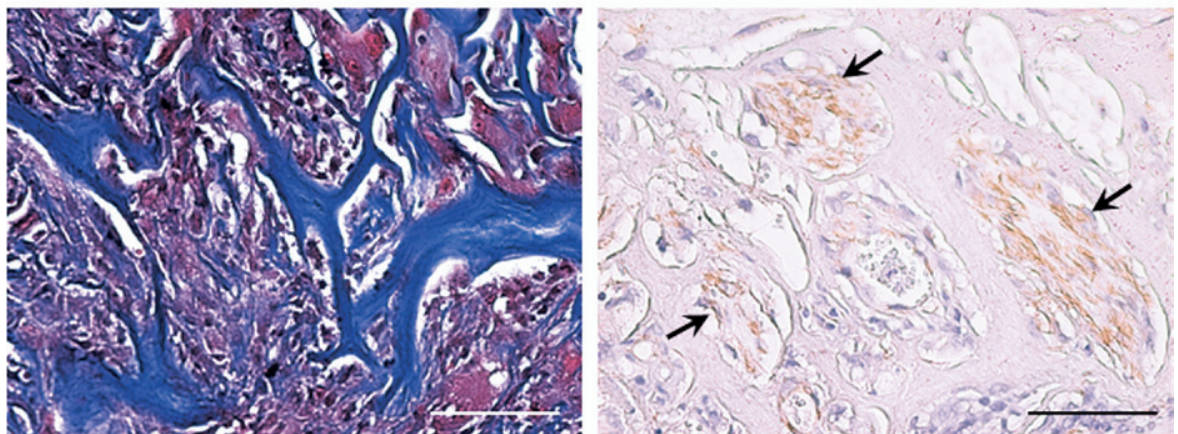

bFGF/MSC Patch
Figure 3. Photomicrographs of the middle layers of the control, bFGF, and bFGF/MSC patches retrieved at 4 weeks postoperatively stained with Masson's trichrome and for $\alpha$-SMA, respectively. The positive stained cells for $\alpha$-SMA is indicated by. Scale bars, $50 \mu \mathrm{m}$.
MSC $\left(552 \pm 25\right.$ vessels $/ \mathrm{mm}^{2}$ ) patches relatively increased. The amounts of the BrdU-labeled cardiomyocytes $(35 \pm 4$ cells $\left./ \mathrm{mm}^{2}\right), \mathrm{SMCs}\left(79 \pm 6\right.$ cells $\left./ \mathrm{mm}^{2}\right)$, and endothelial cells $\left(25 \pm 4\right.$ cells $\left./ \mathrm{mm}^{2}\right)$ stayed approximately the same as those observed at 4 weeks postoperatively. There were still no cardiomyocytes seen in the control and bFGF patches.

\section{Discussion}

Recently, bone marrow-derived MSCs have gained attention for their therapeutic potential. Under differentiationinducing culture conditions, MSCs have the capacity to differentiate into other cell types. ${ }^{3}$ During myogenic differentiation, MSCs continued to proliferate and readily formed myotubes. Additionally, the results obtained in the flow cytometric analysis showed that the induced MSCs stained positively for cardiac-specific troponin $\mathrm{T}$.

At retrieval, it was found that none of the patches thinned or dilated throughout the entire course of the study. This indicated that the mechanical strength of each of the studied groups was strong enough to tolerate the right ventricular pressure of the animal and prevented the implanted patch from dilation.

Host cells together with neoconnective tissue fibrils and neomicrovessels were clearly observed in the outer (epicardial) layers of the control, bFGF, and bFGF/MSC patches, indicating that the outer layers of all studied groups became well integrated with their host tissues (Figure E3). Additionally, an intact layer of neomesothelial cells, resting on 


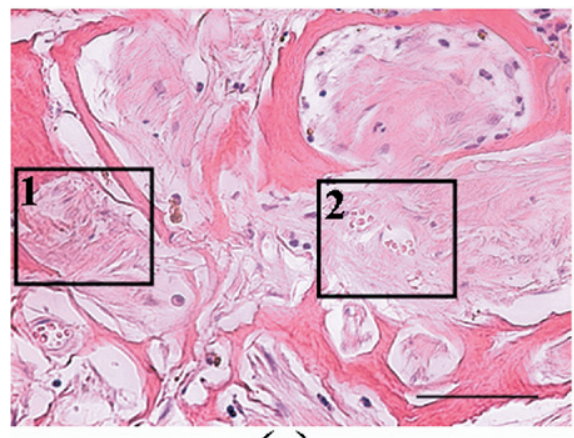

(a)

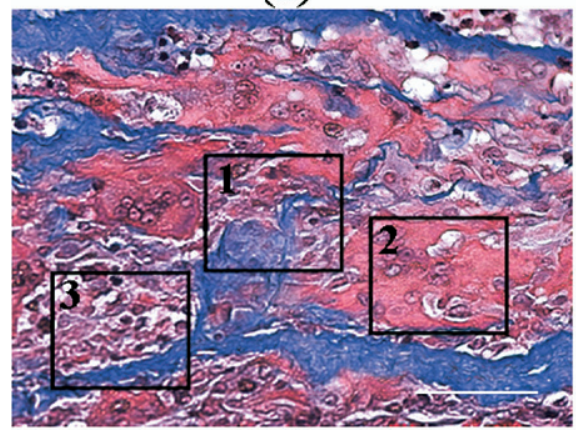

(d)

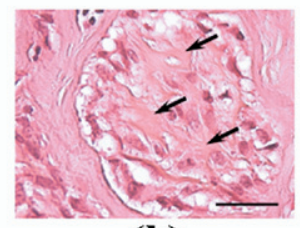

(b)

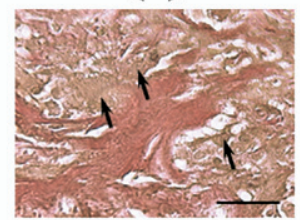

(e)

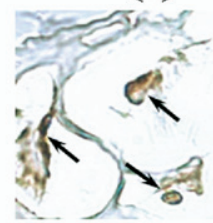

(g)

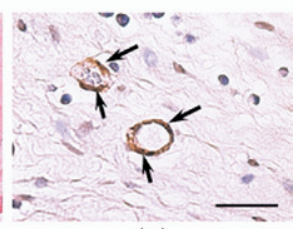

(c)

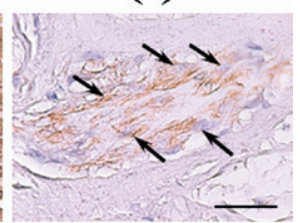

(f)

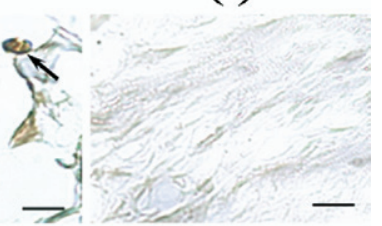

(h)
Figure 4. Photomicrographs of the middle layer $\rightarrow$ of the bFGF/MSC patch retrieved at 12 weeks postoperatively (a) stained with H\&E; (b and c) the insets 1 and 2 of (a) showed the additional staining with Safranin-0 or for factor VIII; (d) stained with Masson's trichrome; (e, $f$, and $g$ ) the insets 1, 2, and 3 of (d) showed the additional staining with EVG for $\alpha$-SMA and double-immunostaining for $\alpha$-sacromeric actin (blue) and anti-BrdU (brown); (h) the negative data for the bFGF patch retrieved at 12 weeks postoperatively. Scale bars, $50 \mu \mathrm{m}$ (a and b), $30 \mu \mathrm{m}$ (b, c, e, and f), and $10 \mu \mathrm{m}$ (g and h). the regenerated tissues, was identified by the van Gieson stain (Figure 2). Several authors have suggested that multipotential cells present within the connective matrix can differentiate into mesothelial cells and contribute to surface reepithelialization. ${ }^{21}$ Remesothelialization on the outer surface of each implanted patch is assumed to play an important role in the prevention of postsurgical adhesions (Figure E2). Whitaker and associates ${ }^{22}$ have documented that mesothelial cells prevent adhesions. It is known that the epicardium forms the outer covering of the heart and has an external layer of flat mesothelial cells. ${ }^{23}$

An intimal thickening covered with endothelial cells was found on the inner (endocardial) surfaces of patches from all studied groups. This finding suggested that host endocardial endothelial cells or endothelial progenitor cells were involved in the endothelialization of the inner surface of each implanted patch and prevented it from thrombus formation (Figure E2). ${ }^{24}$ Endothelialization is one of the most promising mechanisms to reduce thrombogenicity of any cardiovascular implants. $^{25}$

Regenerated muscle fibers (with some collagen fibrils), GAGs, and microvessels were observed in the middle layers of patches of all studied groups (Figure 3). The extent of tissue regeneration in the $\mathrm{bFGF}$ and $\mathrm{bFGF} / \mathrm{MSC}$ patches was more pronounced than that in the control patch throughout the entire course of the study. This may be attributed to the fact that the densities of neomicrovessels observed in the bFGF and bFGF/MSC patches were significantly greater than that seen in the control patch.
Delivery of potent angiogenic factors, such as bFGF, from the implanted ECMs may allow vascularization to be enhanced. ${ }^{9}$ It is known that site-specific delivery of angiogenic molecules may provide an efficient means of stimulating localized vessel formation. Our previous results showed that the GAGs inherent in acellular biologic tissues can be used for the binding, modulation, and sustained release of biologically active bFGF ${ }^{26}$ The binding of bFGF with GAGs is mediated by specific ionic interactions between both 2-O-sulfate groups and N-sulfate groups of GAG molecules and certain lysine and arginine residues on bFGF. $^{27}$ The densities of neomicrovessels observed in the bFGF and bFGF/MSC patches increased relatively with time. This result suggested that the sustained release of bFGF from both patches continued to be effective in enhancing vascularization and in the generation of new tissues.

$\alpha$-SMA positively stained cells were identified in all studied groups (Figure 3), indicating the presence of SMCs. It is known that SMCs permit formation of a muscular tissue (Figure 3, stained red by Massson's trichrome) in addition to collagen formation (stained blue by Massson's trichrome). ${ }^{28}$ These findings suggested that host progenitor cells from the systemic circulation or the surrounding tissue may be relevant to the presence of SMCs in the implanted patches. Previous studies reported that SMC transplantation into myocardial infarct scar tissues improves heart function and prevents ventricle dilatation. ${ }^{29}$ 
The amplitudes of epicardial electrograms observed on the $\mathrm{bFGF}$ and $\mathrm{bFGF/MSC}$ patches were stronger than that seen on the control patch during the entire course of the study, an indication of a better electrical conductance. This may be possibly due to a more pronounced tissue regeneration observed in the 2 former patches. However, we were not able to discern the local electrograms observed on the implanted patches from the hosts' own heart electrical activity.

Despite clear evidences of tissue regeneration, no cardiomyocytes were observed in the control and bFGF patches during the study. It is known that adult cardiomyocytes have essentially no regenerative capacity. ${ }^{5}$ In contrast, a number of cardiomyocytes, which stained positively for $\mathrm{BrdU}$ and $\alpha$-sacromeric actin, were observed in the bFGF/MSC patch at 4 weeks postoperatively (Figure E3). It was reported that the MSCs transplanted into a myocardium environment can express myogenic-specific proteins such as $\alpha$-sacromeric actin. ${ }^{10}$ This result indicated that the induced MSCs, transferred within the patch, can engraft and differentiate into cardiomyocytes.

The amount of cardiomyocytes observed in the bFGF/ MSC patch at 12 weeks postoperatively was comparable to that seen at 4 weeks postoperatively. This implied that the cardiomyocytes differentiated from MSCs survived throughout the entire course of the study. It was reported that vascularization, induced mainly by the loaded bFGF in the present study as described above, is important for the longterm survival of the transplanted cells. ${ }^{10}$ It is known that oxygen supply is often limited in a tissue-engineering ECM. Cells more than approximately $200 \mu \mathrm{m}$ from a blood vessel suffer from hypoxia and die. ${ }^{30}$ In tissue repair or regeneration, the vasculature of the host penetrates macroporous matrices utilized for cell delivery via the process of vascularization.

The echocardiographic results showed that the bFGF/ MSC patch was akinetic, despite the presence of cardiomyocytes. This may be attributed to the fact that there were not enough cardiomyocytes present in the bFGF/MSC patch. In our in vitro study, it was found that after BrdU labeling, the proliferation of MSCs were significantly slowed. Additionally, there was no evidence that the implanted MSCs engaged into functional bonds with host cardiomyocytes, which were functional and contractile. These facts are the primary limitations of the present study.

\section{Conclusions}

The study proposed utilization of a bFGF-loaded porous acellular bovine pericardium populated with MSCs as a patch to repair a myocardial defect. Vascularization together with cardiomyocytes differentiated from MSCs and neomuscle fibers were clearly observed in the patch, an indication of tissue regeneration. These observations indicated that the developed patch improved the limited efficacy of currently available cardiac patches in the long-term results.

\section{References}

1. Pasumarthi KB, Field LJ. Cardiomyocyte cell cycle regulation. Circ Res. 2002;90:1044-54.

2. Yoon YS, Lee N, Scadova H. Myocardial regeneration with bonemarrow-derived stem cells. Biol Cell. 2005;97:253-63.

3. Pittenger MF, Mackay AM, Beck SC, Jaiswal RK, Douglas R, Mosca JD, et al. Multilineage potential of adult human mesenchymal stem cells. Science. 1999;284:143-7.

4. Minguell JJ, Erices A, Conget P. Mesenchymal stem cells. Exp Biol Med. 2001;226:507-20.

5. Orlic D, Kajstura J, Chimenti S, Jakoniuk I, Anderson SM, Li B, et al. Bone marrow cells regenerate infracted myocardium. Nature. 2001; 410:701-5.

6. Ye L, Haider HKh, Sim EK. Adult stem cells for cardiac repair: a choice between skeletal myoblasts and bone marrow stem cells. Exp Biol Med. 2006;231:8-19.

7. Chang Y, Chen SC, Wei HJ, Wu TJ, Liang HC, Lai PH, et al. Tissue regeneration observed in a porous acellular bovine pericardium used to repair a myocardial defect in the right ventricle of a rat model. J Thorac Cardiovasc Surg. 2005;130:705-11.

8. Tabata Y. Tissue regeneration based on growth factor release. Tissue Eng. 2003;9:S5-15.

9. Perets A, Baruch Y, Weisbuch F, Shoshany G, Neufeld G, Cohen S. Enhancing the vascularization of three-dimensional porous alginate scaffolds by incorporating controlled release basic fibroblast growth factor microspheres. J Biomed Mater Res A. 2003;65:489-97.

10. Tomita S, Li RK, Weisel RD, Mickle DA, Kim EJ, Sakai T, et al. Autologous transplantation of bone marrow cells improves damaged heart function. Circulation. 1999;100(19 suppl):II247-56.

11. Krupnick AS, Kreisel D, Engels FH, Szeto WT, Plappert T, Popma $\mathrm{SH}$, et al. A novel small animal model of left ventricular tissue engineering. J Heart Lung Transplant. 2002;21:233-43.

12. Wakitani S, Saito T, Caplan AI. Myogenic cells derived from rat bone marrow mesenchymal stem cells exposed to 5-aza-cytidine. Muscle Nerve. 1995; 18:1417-26.

13. Wei HJ, Linag HC, Lee MH, Huang YC, Chang Y, Sung HW Construction of varying porous structures in acellular bovine pericardia as a tissue-engineering extracellular matrix. Biomaterials. 2005 26:1905-13.

14. Chang Y, Lee MH, Liang HC, Hsu CK, Sung HW. Acellular bovine pericardia with distinct porous structures fixed with genipin as an extracellular matrix. Tissue Eng. 2004;10:881-92.

15. Chang Y, Lai PH, Wang CC, Chen SC, Chang WC, Sung HW. Mesothelium regeneration on acellular bovine pericardia loaded with an angiogenic agent (ginsenoside $\mathrm{Rg}_{1}$ ) successfully reduces postsurgical pericardial adhesions. J Thorac Cardiovasc Surg. 2006;132: 867-74.

16. Ozawa T, Mickle DA, Weisel RD, Koyama N, Ozawa S, Li RK. Optimal biomaterial for creation of autologous cardiac grafts. Circulation. 2002;106(12 suppl 1):I176-82.

17. Chiang HK, Chu CW, Chen GY, Kuo CD. A new 3-D display method for 12-lead ECG. IEEE Trans Biomed Eng. 2001;48:1195-202.

18. Miyagawa S, Sawa Y, Taketani S, Kawaguchi N, Nakamura T, Matsuura $\mathrm{N}$, et al. Myocardial regeneration therapy for heart failure hepatocyte growth factor enhances the effect of cellular cardiomyoplasty. Circulation. 2002;105:2556-61.

19. Prophet EB, Mills B, Arrington JB, et al. Laboratory Methods in Histotechnology. 2nd ed. Washington: American Registry of Pathology; 1994

20. Bader A, Schilling T, Teebken OE, Brandes G, Herden T, Steinhoff G et al. Tissue engineering of heart valves-human endothelial cell seeding of detergent acellularized porcine valves. Eur J Cardiothorac Surg. $1998 ; 14: 279-84$. 
21. Leak LV, Ferrans VJ, Cohen SR, Eidbo EE, Jones M. Animal model of acute pericarditis and its progression to pericardial fibrosis and adhesions: Ultrastructural studies. Am J Anat. 1987;180:373-90.

22. Whitaker D, Papadimitriou JM, Walters M. The mesothelium: its fibrinolytic properties. J Pathol. 1982;136:291-9.

23. Macchi E, Cavalieri M, Stilli D, Musso E, Baruffi S, Olivetti G, et al. High-density epicardial mapping during current injection and ventricular activation in rat hearts. Am J Physiol. 1998;275:H1886-97.

24. Ozawa T, Mickle DA, Weisel RD, Koyama N, Wong H, Ozawa S, et al. Histologic changes of nonbiodegradable and biodegradable biomaterials used to repair right ventricular heart defects in rats. $J$ Thorac Cardiovasc Surg. 2002;124:1157-64.

25. Schneider PA, Hanson SR, Price TM, Harker LA. Confluent durable endothelialization of endarterectomized baboon aorta by early attachment of cultured endothelial cells. J Vasc Surg. 1990;11:365-72.
26. Lai PH, Chang Y, Chen SC, Wang CC, Liang HC, Chang WC, et al. Acellular biological tissues containing inherent glycosaminoglycans for loading bFGF promote angiogenesis and tissue regeneration. Tissue Eng. 2006;12:2499-508.

27. Eriksson AE, Cousens LS, Weaver LH, Matthews BW. Threedimensional structure of human basic fibroblast growth factor. Proc Natl Acad Sci U S A. 1991;88:3441-5.

28. Shinoka T, Ma PX, Shum-Tim D, Breuer CK, Cusick RA, Zund G, et al. Tissue-engineered heart valves: autologous valve leaflet replacement study in a lamb model. Circulation. 1996;94(9 Suppl):II164-8.

29. Li RK, Jia ZQ, Weisel RD, Mickle DA, Zhang J, Mohabeer MK, et al. Cardiomyocyte transplantation improves heart function. Ann Thorac Surg. 1996;62:654-60.

30. Carmeliet P, Jain RK. Angiogenesis in cancer and other diseases. Nature. 2000;407:249-57. 


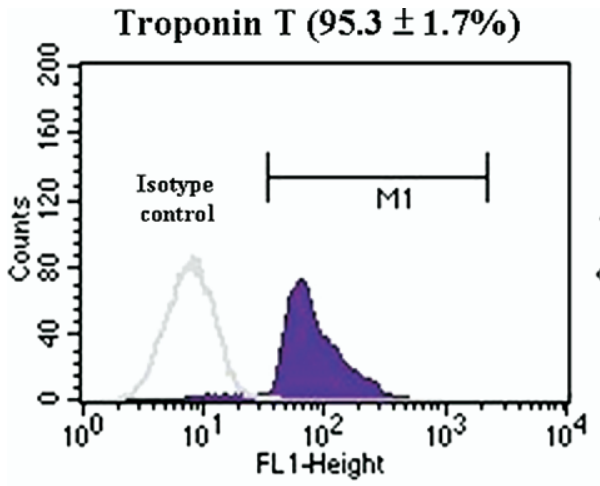

(a)

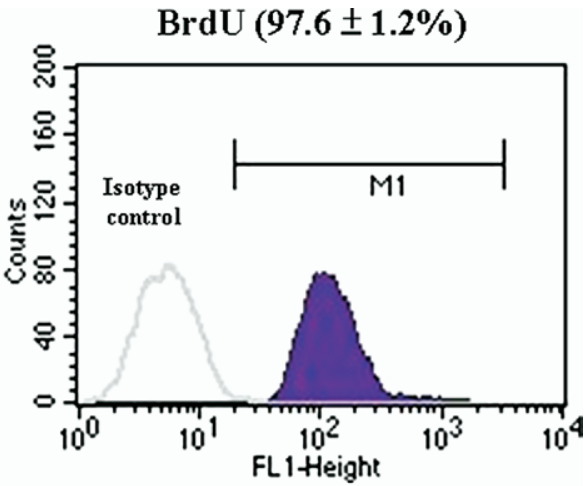

(b)

Figure E1. Representative results from 5 independently performed experiments of flow cytometric analysis of (a) 5-aza-induced MSCs and (b) BrdU-labeled MSCs. 


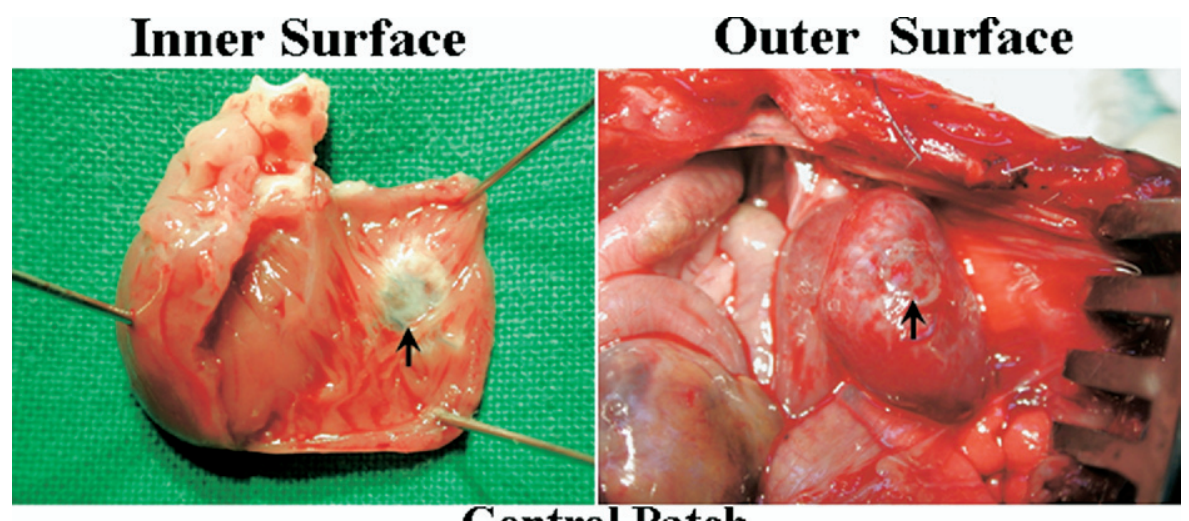

\section{Control Patch}

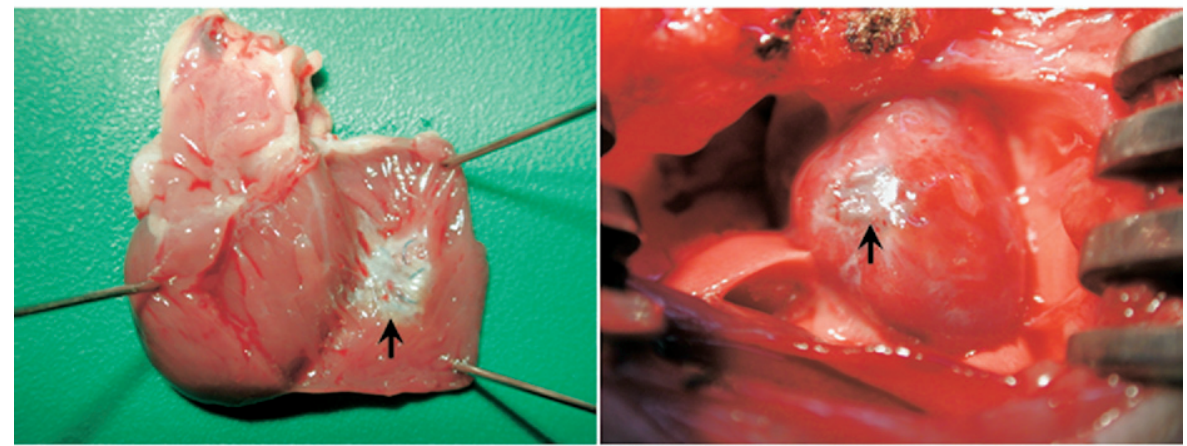

\section{bFGF Patch}

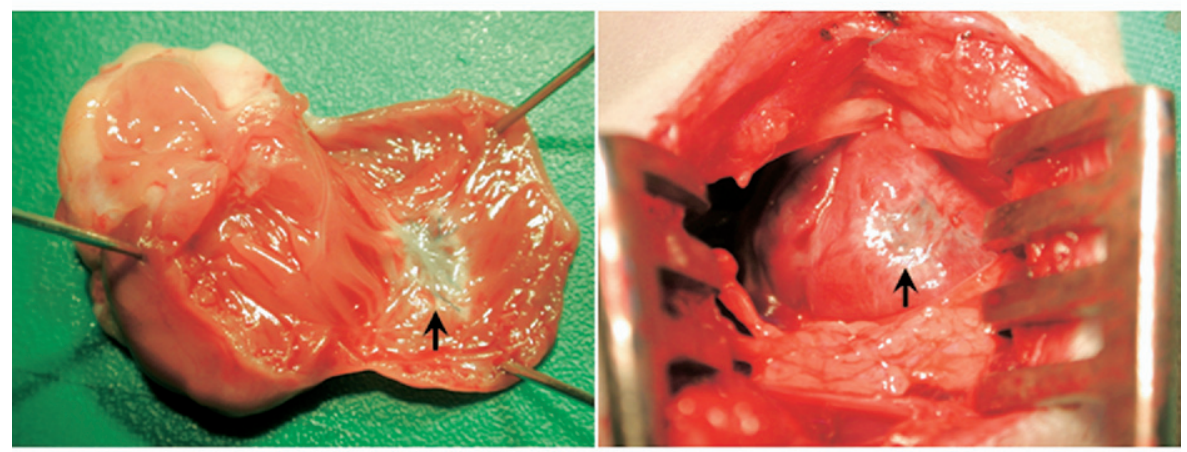

\section{bFGF/MSC Patch}

Figure E2. Photographs of the inner (endocardial) and outer (epicardial) surfaces of the control, bFGF, and bFGF/MSC patches retrieved at 12 weeks postoperatively. The implanted patch is indicated by arrow. $\rightarrow$ 


\section{Inner Surface}

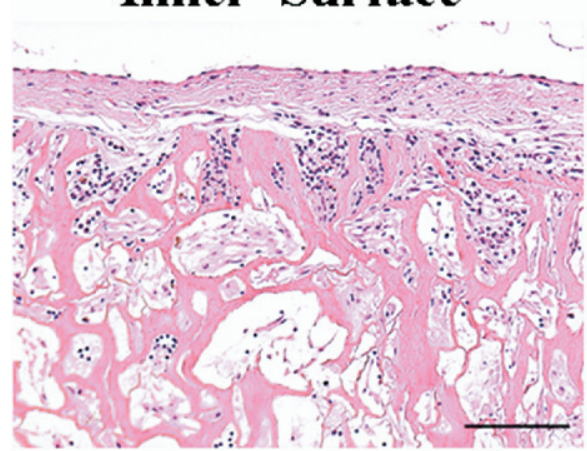

\section{Control Patch}
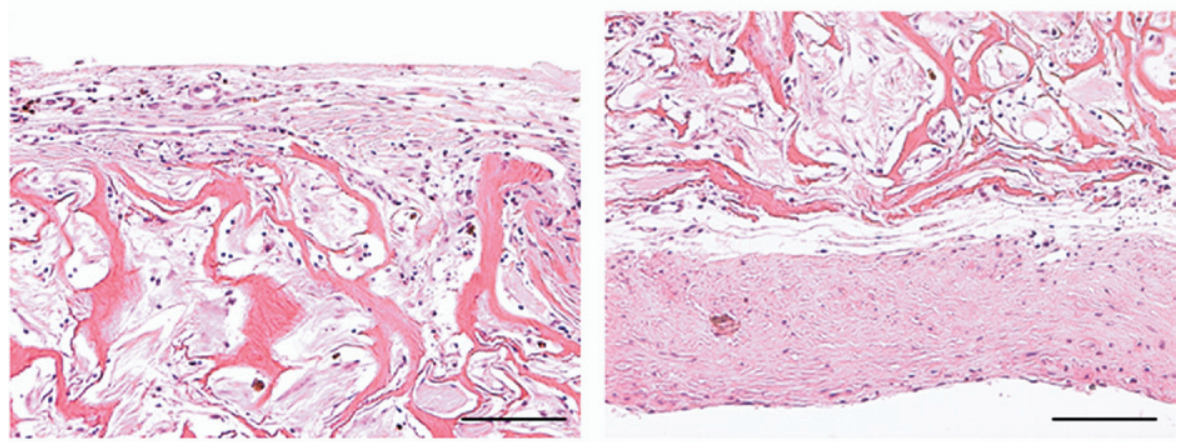

\section{bFGF Patch}
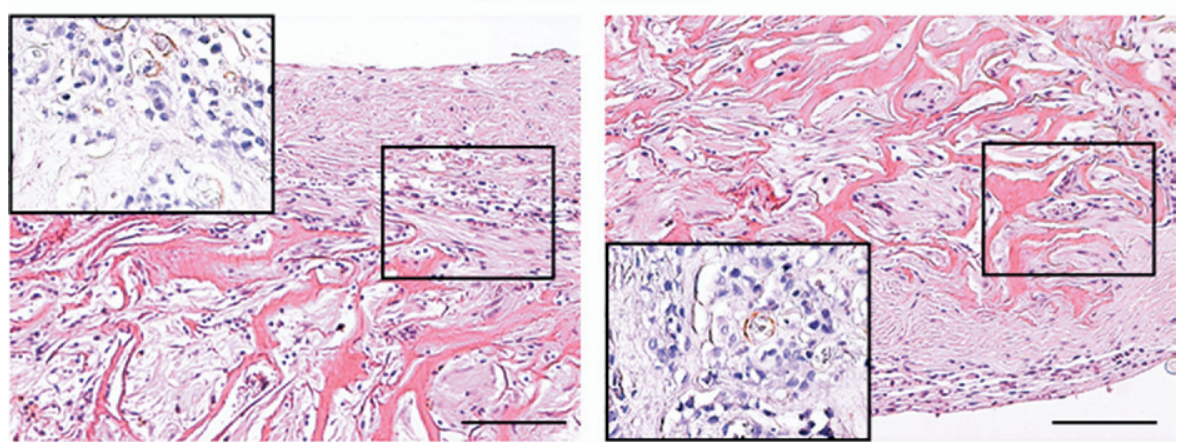

\section{bFGF/MSC Patch}

Figure E3. Photomicrographs of the inner (endocardial) and outer (epicardial) layers of the control, bFGF, and bFGF/MSC patches retrieved at 4 weeks postoperatively stained with H\&E; insets of the bFGF/MSC patch magnified an additional fourfold photomicrographs that stained for factor VIII. Scale bars, $100 \mu \mathrm{m}$. 


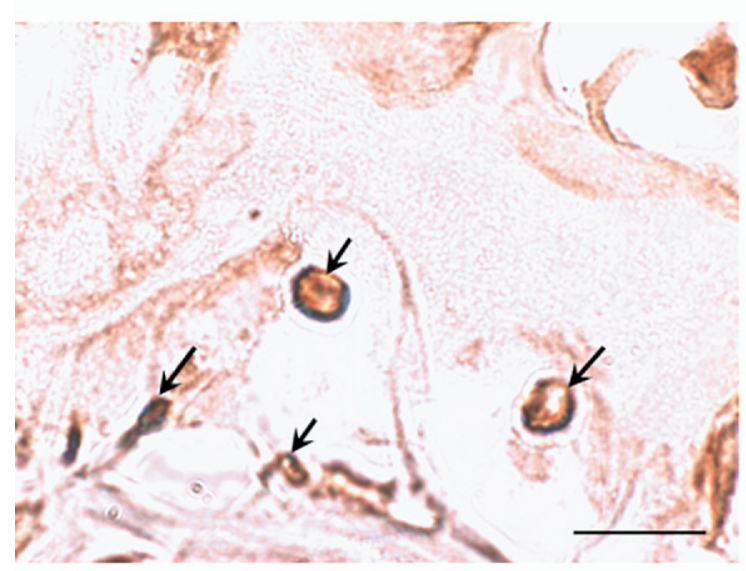

(a)

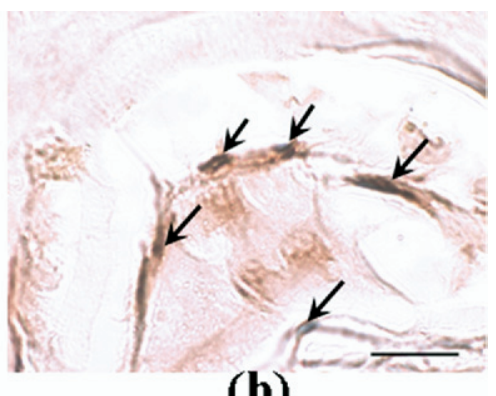

(b)

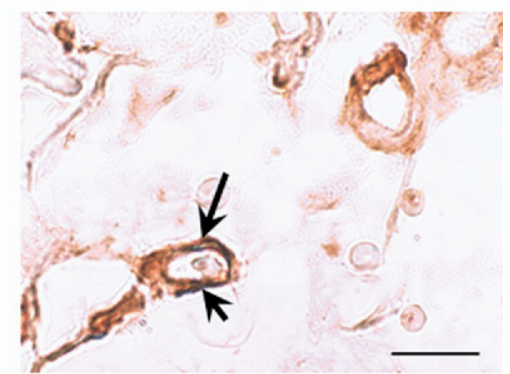

(c)

Figure E4. Photomicrographs of the bFGF/MSC patch retrieved at 4 weeks postoperatively double-immunostained for: (a) $\alpha$-sacromeric actin (blue) and anti-BrdU (brown); (b) $\alpha$-SMA (blue) and anti-BrdU (brown); (c) factor VIII (blue) and anti-BrdU (brown). Scale bars, $10 \mu \mathrm{m}$. 